\title{
Management of Multi-Drug Resistant Methicillin Resistant Staphylococcus aureus Induced Pneumonia with New Antibiotic Adjuvant Entity: A Retrospective Study
}

\author{
Mohammad Shameem \\ Department of Tuberculosis and Chest Disease, JN Medical College, Aligarh Muslim University, Aligarh, India \\ Email: shameem.100@rediffmail.com
}

Received 1 September 2015; accepted 26 October 2015; published 29 October 2015

Copyright (C) 2015 by author and Scientific Research Publishing Inc. This work is licensed under the Creative Commons Attribution International License (CC BY). http://creativecommons.org/licenses/by/4.0/

(c) (i) Open Access

\begin{abstract}
Aim/Objective: Increase in incidences of pneumonia due to multi-drug resistant methicillin resistant Staphylococcus aureus (MRSA) in both community and health care settings is of great concern globally. Present study aims to retrospectively analyze the efficacy of new fixed dose combination with antibiotic adjuvant entity (FDC) in comparison with vancomycin to treat patients with multidrug resistant MRSA pneumonia. Materials and Methods: During this retrospective study, case sheets of patients who were treated for MRSA pneumonia with vancomycin or fixed dose combination of vancomycin + ceftriaxone + adjuvant (FDC) between 20 March 2010 to 20 October 2014 at tertiary care center, were analyzed. Various demographic features, antibiotic therapy, length of treatment duration and the resulting efficacy were evaluated. Microbiological success was measured in terms of bacterial eradication, while clinical success was monitored in terms of complete omission of systemic signs and symptoms. Results: Among 136 patients analyzed, 113 cases were having positive culture for MRSA, and hence were further analyzed. Out of these 113 patients, empirical treatment with vancomycin was given in 59 patients and 54 patients were treated with FDC empirically. After initial culture reports, 22 patients showing resistance to vancomycin were shifted to FDC. Amidst all the patients, 24 (64.86\%) of 37 from vancomycin group and $62(81.57 \%)$ of 76 from FDC group achieved clinical success. 9 patients out of these failure cases were cured with FDC + colistin combination therapy. Failure rates in FDC treated patients were significantly low $(6.57 \%)$ as compared to vancomycin group $(13.51 \%)$. Conclusion: For the treatment of different types of multi-drug resistant MRSA pneumonia, the empirical intravenous FDC therapy was safe and well tolerated with higher efficacy than vancomycin. Most of the vancomycin failure cases responded to FDC therapy and were cured. This retrospective study also concludes that an alternative option of FDC + colistin is safe and effective to treat the patients which fail to respond to FDC monotherapy.
\end{abstract}

How to cite this paper: Shameem, M. (2015) Management of Multi-Drug Resistant Methicillin Resistant Staphylococcus aureus Induced Pneumonia with New Antibiotic Adjuvant Entity: A Retrospective Study. International Journal of Clinical Medicine, 6, 784-795. http://dx.doi.org/10.4236/ijcm.2015.610104 


\section{Keywords}

\section{Pneumonia, MRSA, Multi-Drug Resistant Bacteria, Fixed Dose Combination, Retrospective Study}

\section{Introduction}

Pneumonia is one of the most common infections responsible for hospitalization. Until recently, though it was uncommon and infrequent cause of nosocomial pneumonia [1], in the past decade, MRSA was among the most frequently identified pathogen causing different types of pneumonia worldwide [2] [3]. In fact MRSA now accounts for $20 \%-40 \%$ of pneumonia cases [1].

According to American Thoracic Society, currently accepted classifications of pneumonia include community-acquired pneumonia (CAP), hospital-acquired pneumonia (HAP), health care associated pneumonia (HCAP) and ventilator-associated pneumonia (VAP) [4]. HCAP includes those patients who were hospitalized in an acute care hospital for two or more days within 90 days of the infection residing in a nursing home or long-term care facility with intravenous antibiotic therapy. HAP is defined as pneumonia that occurs within 48 to 72 hrs after hospital admission and that was not present at the time of admission. VAP refers to pneumonia that occurs 48 hours or more after endotracheal intubation [4]. Pneumonia developing outside of the hospital setting has traditionally been classified as community-acquired pneumonia (CAP) [5]. Epidemiological studies indicate that the prevalence of community-associated (CA)-MRSA infections is rising, though most involve skin and soft tissue infections [6] [7]. While the actual incidence of MRSA in community acquired pneumonia remains low, the incidence of MRSA in healthcare-associated pneumonia (HCAP) accounts for $28 \%$ of health care-associated cases [8]. MRSA accounts for 10\% - 40\% cases of HCAP, HAP and VAP [1] [3] [5] [9] [10]. In United States, HAP is the second most common nosocomial infection and its risk increases 6 - 21 folds in patients receiving mechanical ventilation [11]-[14]. It occurs at a rate of 5 - 10 cases per 1000 hospital admissions and accounting $15 \%$ among all hospital acquired infections [11] [15]. Regardless of the type and setting, MRSA pneumonia is associated with significant morbidity and mortality [16] [17]. HAP carries a crude mortality rate of $30 \%$ to $70 \%$ with an estimated attributable mortality rate to pneumonia between $27 \%$ and $50 \%$ [4].

Several studies [18]-[21], demonstrated that MRSA infections were associated with prolonged hospitalizations, increased mortality and increased costs as compared with infections due to methicillin susceptible $S$. $a u$ reus. Along with these, reports [22] [23] have also suggested that in-appropriate and/or delayed antimicrobial therapy to patients with MRSA pneumonia results in increased mortality rates. Hence, it is very essential to assess the type of pneumonia and start with appropriate antimicrobial therapy empirically. Sadly, there are only few therapeutic options for the treatment of patients with MRSA infections. The primary option is intravenous vancomycin therapy which is the only antibiotic available for the treatment of MRSA pneumonia, because other antimicrobials including the fluoroquinolones and third generation cephalosporins are ineffective against MRSA [24]. Unfortunately, the cure rate for vancomycin has been disappointing [25]-[27] with high mortality rates [25]. However, comparatively decreased mortality cases were reported by Wunderink et al. [28] in a clinical trial study and attributed the decreased mortality rates to the optimized vancomycin dosing and overall improvement in the quality of care in patients with MRSA pneumonia. The decreased cure rates with vancomycin may be due to any previously reported reasons [24] [29]-[32], which can limit the usefulness of vancomycin monotherapy [33] [34]. All these aspects accentuate the need for new antibiotics. A new antibiotic adjuvant entity of ceftriaxone + vancomycin is increasingly being used in Indian hospitals. Various reports of the in-vitro susceptibility studies [35]-[39] hint the possibility of this new FDC to overcome the hurdles of MRSA infections clinically. If effective in-vivo clinical success is achieved by this new FDC, it can be a potent alternative to vancomycin to treat infections caused by the multi-drug resistant MRSA strains. In view of the above aspects and to address these issues, a retrospective study was planned to analyze the case records of the multi-drug resistant MRSA pneumonia patients treated with vancomycin and FDC.

\section{Materials and Methods}

\subsection{Study Design Overview}

This study was a retrospective, observational analysis of the data collected from tertiary care hospital from years 
2010 to 2014. The present study was carried out in accordance with the ethical principles of the Declaration of Helsinki and to the current norm for observational studies. Due to the retrospective study design, informed consent was not deemed necessary.

\subsection{Patient Selection}

The patients were selected by going through their case history sheets of the hospitals. Hospitalized patients aged above 18 years, who were admitted between March-2010 and October-2014 were considered for the study. The other criteria for patients inclusion were; 1) patients with final diagnosis of HAP, HCAP, CAP or VAP based on the clinical investigations and relevant signs and symptoms, 2) patients with baseline culture specimen positive for MRSA and 3) patients in whom either vancomycin or FDC were used empirically.

\subsection{Patient Analysis}

Case history sheets of all the patients were reviewed and relevant informations like patient age, gender, comorbidities, microbiology tests, antibiotic therapy, dose and duration, switch to the second line or considering additional cover and the reasons for the shift/additional cover and length of the hospital stay were recorded. Among all the case sheets analyzed, 113 patients which were given either vancomycin and FDC and fulfilled the other above mentioned inclusion criteria were included for the analysis. The antibiotic doses used in the therapy were $1 \mathrm{~g} / 12 \mathrm{hrs}, 1.5 \mathrm{~g} / 12 \mathrm{hrs}$ for vancomycin and FDC respectively. For colistin therapy, a loading dose of 9 MIU followed by BD doses of 4.5 MIU were used.

\subsection{Initial Antibiotic Treatment and Outcomes}

For better presentation and easy understanding (Figure 1), the patients analyzed retrospectively were broadly divided in to two groups; Group G1-patients [59 (52.21\%)] to whom vancomycin was administered empirically and Group G2-patients [54 (47.79\%)] which are on empirical FDC therapy. Out of 59 patients from group G1, 37 (62.71\%) patients (Group G1A) were diagnosed with pathogens susceptible to both vancomycin and FDC, where as remaining 22 (37.29\%) patients were infected with cultures resistant to vancomycin and

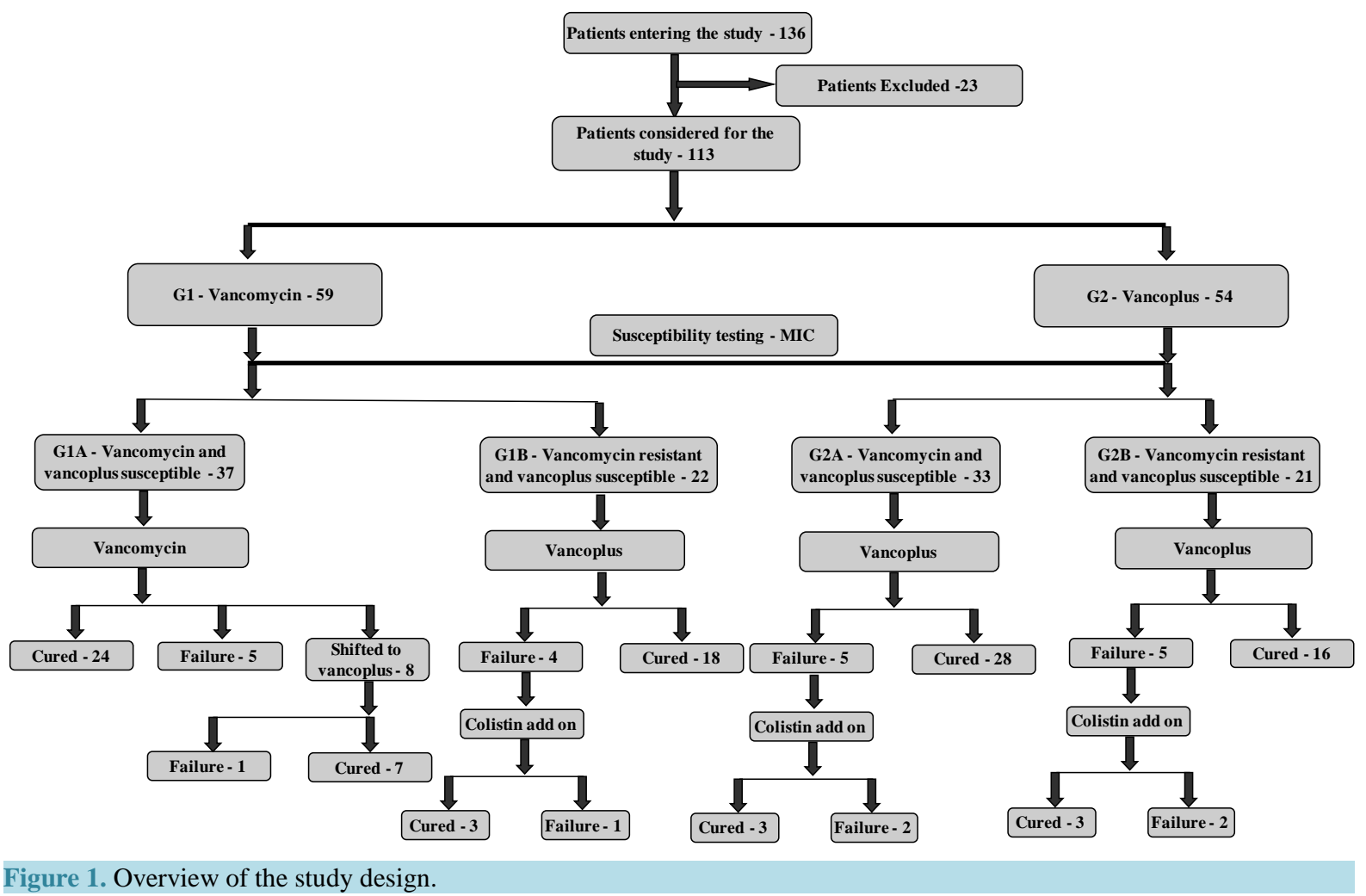


susceptible to FDC and hence were shifted to FDC therapy. Out of 54 patients from group G2, 33 (61.11\%) patients (Group G2A) were with vancomycin and FDC susceptible culture infection and the remaining 21 (38.89 \%) patients were identified with cultures susceptible to FDC and resistant to vancomycin. The progress of the therapy was measured in terms of clinical improvement in signs and symptoms. After the initial assessment (after 2/3 days) of the clinical progress (signs of recovery), the decision on whether to continue with empirical therapy or shift to other regime was taken. The patients (from Group G1A) showing improvement with empirical vancomycin were continued with the same antibiotic and the patients which failed to show any improvement were shifted to FDC. Patients (Group G1B) with infections diagnosed with vancomycin resistant and FDC susceptible culture infection, therapy was shifted to FDC. Among the patients in which FDC therapy was not effective (deterioration) were given an additional cover of colistin. Even though 33 patients from Group G2A were diagnosed with vancomycin susceptible infection, the empirical therapy was not deescalated considering the patients positive response (clinical improvement) to the empirical regimen (FDC).

\subsection{Patient Evaluations and Definitions}

All the Patients enrolled into the study were thoroughly evaluated by examining chest X-rays, culture and sensitivity reports (blood, endo-tracheal cultures, bronch-oalveolar lavage (BAL) specimens), hematology and biochemistry and other relevant investigations. All the investigations carried throughout study period were evaluated to correlate the clinical improvement of patient compared to baseline. All the evaluations were done to derive a co-relation of lab results with clinical parameters. All the lab parameters were tabulated to assess the change in lab parameters during the course of treatment. All the deranged parameters were compared with baseline parameters and finally evaluated for reason of derangement. The lab parameters were also evaluated to rule out any toxicity like nephro-toxicity during course of treatment. Minimum inhibitory concentrations (MICs) of antibiotics for clinical isolates were determined by microbroth dilution method according to the Clinical and Laboratory Standards Institute (CLSI). The assessment of microbiological response at patient level was based on the results of susceptibilities of the isolated pathogens and clinical outcome of the patients. The microbiological response was considered satisfactory/success when the original causative pathogen was completely eradicated or presumed to be eradicated (i.e. when patients were not available for microbial assessment and/or further sampling was not considered significant because of clinical cure/improvement). The response was considered unsuccessful/failure if the diagnosed pathogen persisted or presumed to be persisted or a new pathogen was isolated from the original site of infection during the study (super-infection).

\subsection{Definitions}

Clinical success: The patients response was considered as clinical success when, the patient recovered with either first line empiric antibiotic therapy or a step down from the initial therapy [40].

Clinical failure: An individual case was defined as clinical failure when either the treatment was switched to the second line antibiotics or when the death of patients occurs.

First line/empiric antibiotic therapy: It is defined as the regime started at the beginning before the availability of any cultural data.

Second-line antibiotic therapy: It is defined as the addition of one or more antibiotics to the initial regime or as a complete or partial shift of the initial antibiotic with another parenteral antibiotic regime.

\section{Results}

\subsection{Patients and Demographic Characteristics}

Out of 136 patients considered for the retrospective analysis, 113 patients were confirmed with MRSA pneumonia who met other study entrance criteria and hence were further analyzed (Figure 1). The baseline and demographic characteristics of the patients which were given either vancomycin $(n=59)$ or FDC $(n=54)$ empirically are given in Table 1. Most of the baseline characteristics among the patients from both the groups were comparable. Male population was more when compared to their counter parts in both the groups with male: female ratio of 37:22 and 32:22 for vancomycin and FDC groups respectively. The mean age of patients in vancomycin was $58.47 \pm 14.67$ and the same in patients belonging to FDC group was $59.85 \pm 13.99$. The analysis of the disease severity data measured in terms of APACHE II score reveals that irrespective of the groups, majority of 
Table 1. Baseline and demographic characteristics.

\begin{tabular}{|c|c|c|}
\hline \multirow{2}{*}{ Characteristic } & \multicolumn{2}{|c|}{ Data for patients who received } \\
\hline & Vancomycin & FDC \\
\hline Evaluable patients & 59 & 54 \\
\hline Sex ratio—male:female [n (\%)] & 37:22 (62.71\%:37.29\%) & 32:22 (59.25\%:40.75\%) \\
\hline Age, mean year SD & $58.47 \pm 14.67$ & $59.85 \pm 13.99$ \\
\hline \multicolumn{3}{|l|}{ APACHE II score } \\
\hline$<15$ & $44(74.57 \%)$ & $41(75.92 \%)$ \\
\hline$\geq 15$ & 15 (25.43\%) & $13(24.08 \%)$ \\
\hline \multicolumn{3}{|l|}{ Co-morbidities } \\
\hline Diabetes mellitus & $18(30.50 \%)$ & $19(35.18 \%)$ \\
\hline Cardiac & $27(45.76 \%)$ & $23(42.59 \%)$ \\
\hline Kidney & 7 (11.86\%) & $4(7.40 \%)$ \\
\hline Chronic obstructive pulmonary disease (COPD) & $33(55.93 \%)$ & $35(64.81 \%)$ \\
\hline Neurological disease & $9(15.25 \%)$ & $10(18.51 \%)$ \\
\hline \multicolumn{3}{|l|}{ Pneumonia types } \\
\hline Community associated & $8(13.55 \%)$ & 7 (12.96\%) \\
\hline Health care-associated & $26(44.06 \%)$ & $28(51.85 \%)$ \\
\hline Hospital associated & $22(37.28 \%)$ & $15(27.77 \%)$ \\
\hline Ventilator associated & $31(52.54 \%)$ & 28 (51.85\%) \\
\hline
\end{tabular}

patients were having a score of $<15.44(74.57 \%)$ out of 59 patients from vancomycin group were having APACHE II score of $<15$, whereas 41 (75.92\%) patient out of 54 from FDC group were having the severity score of $<15$. Among different types of pneumonia, there are 54 cases of HCAP (26 in vancomycin group; 28 in FDC group) and 37 cases of HAP (22 in vancomycin group; 15 in FDC group) and 59 cases of VAP (31 in vancomycin group; 28 in FDC group) and the 15 cases were of CAP (8 in vancomycin group; 7 in FDC group). Chronic obstructive pulmonary disease (COPD) was the most common co-morbidity observed in patients from both the groups (33-vancomycin group; 35-FDC group) followed by Cardiac diseases (27-vancomycin group; 23-FDC group), diabetes mellitus (18-vancomycin group; 19-FDC group), neurological diseases (9-vancomycin group; 10-FDC group), and least number of cases were observed with kidney disorders (7vancomycin group; 4-FDC group) (Table 1).

\subsection{Clinical Response}

After retrospectively analyzing the case records of all the patients considered for the study, the clinical response of patients was represented as depicted in Figure 1. The patients from both the groups were further divided in to two groups; first vancomycin and FDC susceptible (Group G1A and G2A) and second vancomycin resistant and FDC susceptible (Group G1B and G2B). Empirical vancomycin treatment with successful clinical response was observed in 24 (64.86\%) patients out of 37 patients from Group G1A. The mean treatment duration among these 24 patients was (9.79 days \pm 1.28 (SD). Among the remaining 13 patients who failed to respond, 8 patients were shifted to FDC (because cultures were susceptible to FDC) and 5 patients showed treatment failure. Among 8 shifted patients, 7 (87.5\%) patients achieved clinical clinical success and one patient showed complete treatment failure. The mean treatment duration for these 7 patients was [10.85 days \pm 0.37 (SD)]. 22 patients from Group G1B, who were on empirical vancomycin treatment were shifted to FDC once the microbial susceptibility data were received. 18 (81.81\%) out of 22 shifted patients achieved clinical success with mean treatment duration of 
[11.38 days $\pm 0.84(\mathrm{SD})]$. For the remaining 4 patients, an additional antibiotic cover (colistin) was given, which cured 3 patients with one patient showed complete treatment failure. On the other hand, 33 patients (Group G2A) who were on FDC empirically, continued with the same therapy even after the receipt of the culture reports (vancomycin and FDC susceptible cultures). Among these 33 patients, 28 (84.84\%) patients showed positive response with the mean treatment duration of [7.64 days \pm 0.67 (SD)]. For the remaining 5 patients colistin was given as an additional therapy, to which 3 patients responded and 2 patients failed to respond. Lastly 16 (76.19\%) among 21 patients from Group G2B which were on FDC therapy achieved clinical success with mean treatment duration of (8.5 days \pm 0.51 (SD)). The remaining 5 patients were given an additional cover of colistin, which could generate positive clinical response in 3 patients and 2 patients did not responded to the therapy.

Clinic response among the subgroups is depicted in Table 2. The clinical response in all the sub groups followed a similar pattern as that of a overall clinical response. In patients who were diagnosed with pneumonia caused by vancomycin and FDC susceptible MRSA, the number of patients cured with FDC empirical therapy were significantly high as compared to vancomycin treated ones. Higher age resulted in lower cure cases (42.85\% for $\geq 76$ years patients) in vancomycin treated groups, where as the age did not have any significant effect on the efficacy of FDC (100\% $\geq 76$ years). Both the groups had lower cure rates in patients with higher APACHE II scores (Vancomycin-50\%; FDC-75\%). Both the empirical vancomycin and FDC treatment had higher cure rates in community associated pneumonia $(80 \% ; 100 \%)$ followed by health-care associated pneumonia, ventilator associated pneumonia and hospital acquired pneumonia. The co-morbidities did not have any significant impact on the clinical cure rates in both groups, though highest cure rates in vancomycin group were observed in patients with COPD (76.19) followed by patients with cardiac diseases and neurological disorders (75\%), diabetes mellitus (72.72\%) and least was observed in patients with kidney related diseases (66.66\%). On the other hand, all the patients from FDC group with neurological disorders and kidney diseases achieved clinical success. High cure rates were also observed in patients with COPD (85.71\%) followed by patients with diabetes mellitus $(80 \%)$ and in patients with cardiac diseases (78.57\%). However, the clinical response in the patients diagnosed with vancomycin resistant MRSA. All the 22 patients who were on vancomycin empirically showed deteriorating conditions and forced to shift to FDC therapy after microbiological susceptibility reports. However, the clinical response among all the subgroups in FDC group followed the same trend as that of patients belonging to Group G2A with slight variations in cure rates among the groups. The detailed clinical response in all the subgroups in line with the therapy regime in given in Table 2.

\subsection{Microbiological Response}

The microbial responses evaluated at the end of the therapy were in line with the clinical responses of patients showed to the respective antibiotics. The microbial response evaluated in patients diagnosed with vancomycin and FDC susceptible cultures is depicted in Figure 2. In vancomycin group, 64.84\% patients had microbiological success (documented or presumed eradication) to empirical vancomycin therapy as compared to $84.84 \%$ of FDC treated patients. Super infection was documented in 5 patients (clinical failure before shift of therapy) out of 12 who had material for microbiological culture or documented persistence of MRSA. Among the remaining 8 patients, 7 patients achieved microbial success (presumed 4; documented 3) and the remaining one patient still persisted with MRSA. In 5 patients (FDC group) with documented persistence of MRSA, 3 patients (presumed 2; documented 1) achieved microbial success and 2 patients were tested positive for MRSA persistence. The microbial response evaluated in patients diagnosed with vancomycin resistant and FDC susceptible cultures is depicted in Figure 3. In vancomycin treated group, no patient achieved microbial success (vancomycin resistant cultures) and hence were shifted to FDC. 18 (81.81\%) out of 22 patients (from vancomycin group) and 16 (76.19\%) out of 21 patients (from FDC group) treated with FDC achieved microbiological success. The remaining patients documented with MRSA persistence were given an additional cover of colistin. Among the 4 cultures from vancomycin group which were given colistin, 3 achieved microbial success, whereas the same treatment given in 5 patients from FDC group resulted in microbial eradication in 3 patients.

\section{Discussion}

Irrespective of the type of pneumonia, S. aureus is one of the major pathogens causing pneumonia. Incidences of pneumonia due to $S$. aureus have been increasing in the past couple of decades as evident through a study reported in National Nosocomial Infection Surveillance (NNIS) system. NNIS analysed over 410,000 isolates and 
Table 2. Clinical success rates at the end of the treatment by patient subgroup.

\begin{tabular}{|c|c|c|c|c|c|c|}
\hline \multirow{3}{*}{ Sub group } & \multicolumn{6}{|c|}{ Success rate [no. of successes/total no. (\%)] for: } \\
\hline & \multicolumn{3}{|c|}{ Vancomycin group } & \multicolumn{3}{|c|}{ FDC group } \\
\hline & $\begin{array}{l}\text { Empirical } \\
\text { therapy }\end{array}$ & $\begin{array}{l}\text { Shifted therapy } \\
\text { (FDC) }\end{array}$ & $\begin{array}{l}\text { Colistin add } \\
\text { on therapy }\end{array}$ & $\begin{array}{l}\text { Empirical } \\
\text { therapy }\end{array}$ & $\begin{array}{l}\text { Shifted } \\
\text { therapy }\end{array}$ & $\begin{array}{l}\text { Colistin add } \\
\text { on therapy }\end{array}$ \\
\hline $\begin{array}{l}\text { Evaluable patients for } \\
\text { efficacy analysis }\end{array}$ & & 59 & & & 54 & \\
\hline \multicolumn{7}{|c|}{ Patients with infections caused vancomycin and FDC susceptible MRSA (vancomycin MIC $\leq 1$ ) } \\
\hline Overall clinical success & & 31/37 (83.78) & & & 1/33 (93.93 & \\
\hline Treatment regime-wise & 24/37 (64.86) & 7/8 (87.5) & $0(0)$ & 28/33 (84.84) & $0(0)$ & $3 / 5(60)$ \\
\hline \multicolumn{7}{|l|}{ Age } \\
\hline$\leq 50$ years & 07/11 (63.63) & $2 / 2(100)$ & $0(0)$ & 6/7 (85.71) & $0(0)$ & $1 / 1(100)$ \\
\hline$\geq 51$ years $\leq 75$ years & 14/19 (73.68) & 4/4 (100) & $0(0)$ & 15/19 (78.94) & $0(0)$ & $2 / 4(50)$ \\
\hline$\geq 76$ years & 03/07 (42.85) & $1 / 2(50)$ & $0(0)$ & 07/07 (100) & $0(0)$ & $0(0)$ \\
\hline \multicolumn{7}{|l|}{ APACHE II score } \\
\hline$<15$ & 19/27 (70.37) & 5/6 (83.33) & $0(0)$ & 22/25 (88) & $0(0)$ & 2/3 (66.66) \\
\hline$\geq 15$ & $5 / 10(50)$ & $2 / 2(100)$ & $0(0)$ & $6 / 8(75)$ & $0(0)$ & $1 / 2(50)$ \\
\hline \multicolumn{7}{|l|}{ Types of pneumonia } \\
\hline Community associated & 04/05 (80) & $1 / 1(100)$ & $0(0)$ & $4 / 4(100)$ & $0(0)$ & $0(0)$ \\
\hline Health-care associated & 13/17 (76.47) & 4/4 (100) & $0(0)$ & 17/21 (80.95) & $0(0)$ & 3/4 (75) \\
\hline Hospital associated & 9/12 (75) & 3/3 (100) & $0(0)$ & 5/7 (71.42) & $0(0)$ & $1 / 2(50)$ \\
\hline Ventilator associated & 12/18 (66.66) & 5 /6 (83.33) & $0(0)$ & 14/17 (82.35) & $0(0)$ & 2/3 (66.66) \\
\hline \multicolumn{7}{|l|}{ Causative pathogen } \\
\hline MRSA only & 22/26 (92.30) & 3/3 (100) & $0(0)$ & 25/27 (92.59) & $0(0)$ & $1 / 2(50)$ \\
\hline $\begin{array}{l}\text { MRSA mixed with other } \\
\text { pathogens }\end{array}$ & 2/11 (18.18) & $4 / 5(80)$ & $0(0)$ & $3 / 6(50)$ & $0(0)$ & 2/3 (66.66) \\
\hline \multicolumn{7}{|l|}{ Type of co-morbidities } \\
\hline Diabetes mellitus & 8/11 (72.72) & 3/3 (100) & $0(0)$ & 08/10 (80) & $0(0)$ & $2 / 2(100)$ \\
\hline Cardiac diseases & $12 / 16(75)$ & 4/4 (100) & $0(0)$ & 11/14 (78.57) & $0(0)$ & 2/3 (66.66) \\
\hline Kidney diseases & 2/3 (66.66) & 1/1 (100) & $0(0)$ & $1 / 1(100)$ & $0(0)$ & $0(0)$ \\
\hline $\begin{array}{l}\text { Chronic obstructive pulmonary } \\
\text { disease (COPD) }\end{array}$ & 16/21 (76.19) & $4 / 5(80)$ & $0(0)$ & 18/21 (85.71) & $0(0)$ & 2/3 (66.66) \\
\hline \multirow[t]{2}{*}{ Neurological disorders } & $3 / 4(75)$ & 1/1 (100) & $0(0)$ & 4/4 (100) & $0(0)$ & $0(0)$ \\
\hline & $0 / 1(0)$ & $1 / 1(100)$ & $0(0)$ & 2/3 (66.66) & $0(0)$ & 1/1 (100) \\
\hline \multicolumn{7}{|c|}{ Patients with infections caused vancomycin resistant and FDC susceptible MRSA (vancomycin MIC $\geq 1$ ) } \\
\hline Overall clinical success & & 21/22 (95.45) & & & 9/21 (90.47) & \\
\hline Treatment regime-wise & $0 / 22(0)$ & $18 / 22(81.81)$ & $3 / 4(75)$ & 16/21 (76.19) & $0(0)$ & $3 / 5(60)$ \\
\hline \multicolumn{7}{|l|}{ Age } \\
\hline$\leq 50$ years & $0 / 10(0)$ & 9/10 (90) & $1 / 1(100)$ & 6/9 (66.66) & $0(0)$ & $1 / 3(33.33)$ \\
\hline$\geq 51$ years $\leq 75$ years & $0 / 8(0)$ & $7 / 8(87.5)$ & 1/1 (100) & 7/9 (77.77) & $0(0)$ & $1 / 2(50)$ \\
\hline$\geq 76$ years & $0 / 4(0)$ & 2/4 (50) & 1/2 (50) & 3/3 (100) & $0(0)$ & $0(0)$ \\
\hline
\end{tabular}




\section{Continued}

\section{APACHE II score}

$<15$

$\geq 15$

Types of pneumonia

Community associated

Health-care associated

Hospital associated

Ventilator associated

Causative pathogen

MRSA only

MRSA mixed with other pathogens

$\begin{array}{cccccc}0 / 19(0) & 17 / 19(89.47) & 1 / 2(50) & 13 / 16(18.75) & 0(0) & 2 / 3(66.66) \\ 0 / 3(0) & 1 / 3(33.33) & 2 / 2(100) & 3 / 5(60) & 0(0) & 1 / 2(50)\end{array}$

0/03 (0)

$$
\text { 03/03 (100) }
$$

$0 / 0(0)$

7/9 (77.77)

$1 / 2(50)$

3/3 (100)

$0(0)$

$0(0)$

$0 / 9(0)$

9/10 (90)

$1 / 1(100)$

6/7 (85.71)

0 (0)

$1 / 2(50)$

$0 / 10$

12/13 (92.30)

1/1 (100)

6/8 (75)

0 (0)

1/1 (100)

0/13 (0)

\section{$14116(87.5)$}

0/16 (0)

14/16 (87.5)

$2 / 2(100)$

9/11 (81.81)

0 (0)

$1 / 2(50)$

$0 / 6(0)$

4/6 (66.66)

$1 / 2(50)$

11/13 (84.61)

$0(0)$

$1 / 2(50)$

Type of co-morbidities

Diabetes mellitus

Cardiac

Kidney

Chronic obstructive pulmonary disease (COPD)

Neurological disease

$0 / 7(0)$

6/7 (85.71)

1/1 (100)

7/9 (77.77)

$0(0)$

2/2 (100)

0/11 (0)

9/11 (81.81)

$1 / 2(50)$

$0 / 4(0)$

4/4 (100)

$0(0)$

8/9 (88.88)

$0(0)$

0 (0)

$0 / 12(0)$

10/12 (83.33)

$1 / 2(50)$

2/3 (66.66)

$0(0)$

1/1 (100)

$0 / 5(0)$

$4 / 5$ (80)

1/1 (100)

11/14 (78.57)

$0(0)$

1/3 (33.33)

Bacteremia

$0 / 3(0)$

$1 / 1(100)$

5/6 (83.33)

$0(0)$

1/1 (100)

Bactorata

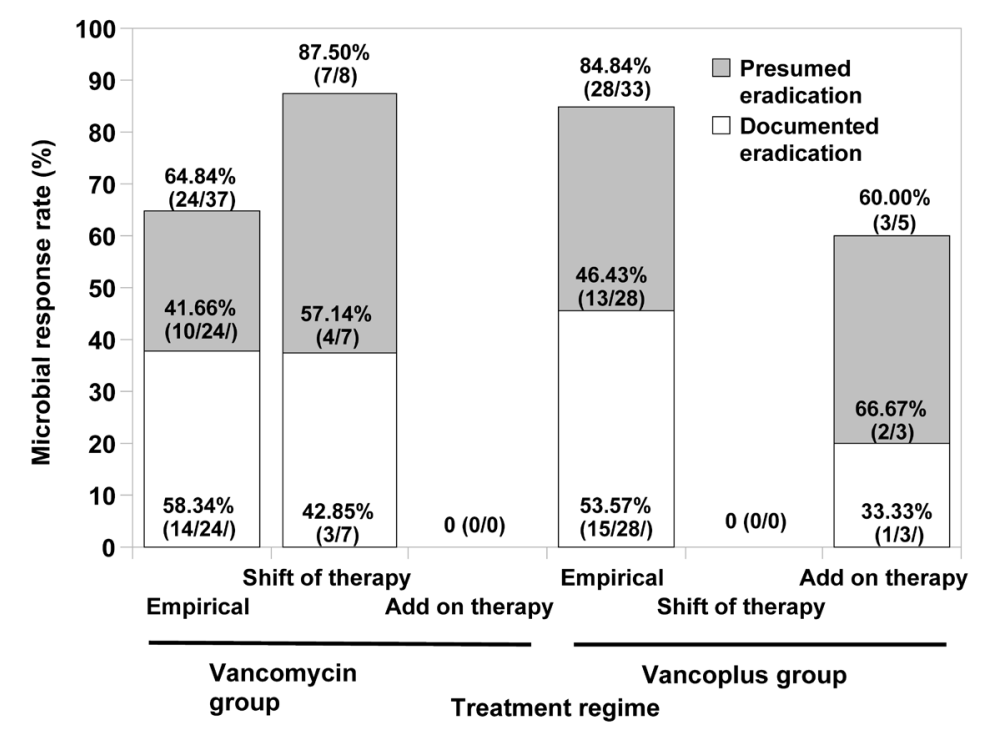

Figure 2. Microbial response rates in patients with vancomycin and FDC susceptible cultures.

reported that rates of $S$. aureus pneumonia cases had alarmingly increased from $13.4 \%$ in 1975 to $27.80 \%$ in 2003 [41]. Despite the implementation of effective infection prevention and control measures in some countries [42] [43] (which resulted in some decrease in MRSA infections), the burden of the MRSA respiratory infections remains alarming. Approximately 36,540 pneumonias attributed to MRSA were estimated to have occurred in 


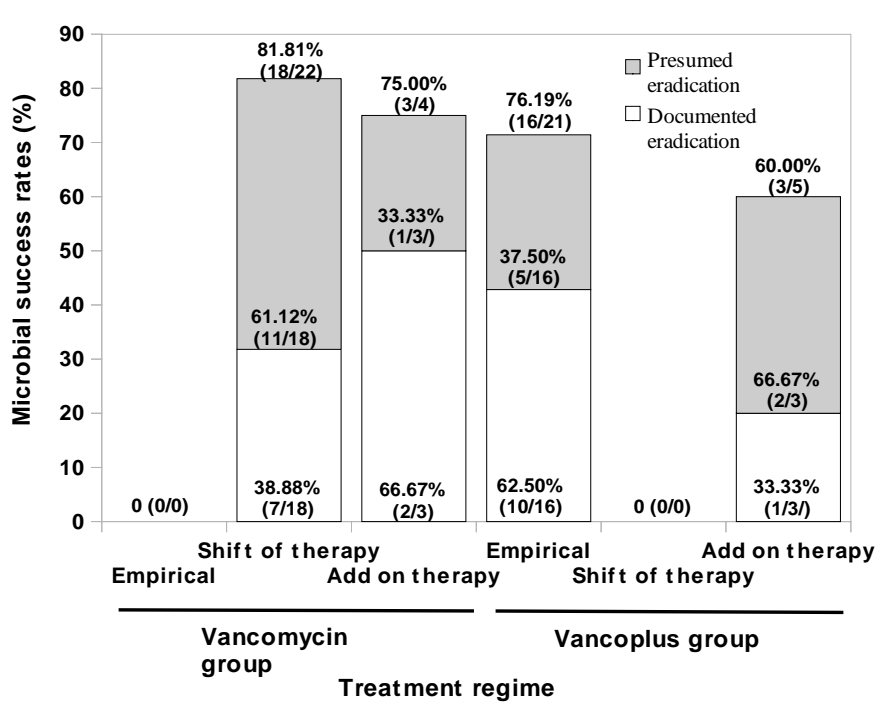

Figure 3. Microbial response rates in patients with Vancomycin resistant and FDC susceptible cultures.

US hospitals in 2005 [7]. In a study conducted in over 4500 pneumonia patients with documented culture positive reports, the most predominant pathogen was $S$. aureus. Among these patients MRSA accounted in 8.9, 49 and $14.6 \%$ patients with CAP, HAP and VAP, respectively [3]. In the study presented here, we come out with the retrospective analysis of data sheets for 113 patients diagnosed with different pneumonia like CAP, HCAP, HAP and VAP and treated with either vancomycin or FDC empirically.

Depending on the susceptibility of the causative pathogen, the available treatment options for different pneumonia include 7 to 21 days of intravenous vancomycin, linezolid or clindamycin [44]. Vancomycin has been considered to be the reference standard for the treatment of invasive MRSA infections, as a result of its relatively clean safety profile, its durability against the development of resistance, and, for many years, the lack of other approved alternatives. In the present study, the results of the efficacy analysis for the antibiotics revealed that the clinical cure rates among vancomycin treated patients (G1A) was $64.86 \%$ with 13 (35.14\%) patients failed to respond to the empirical vancomycin therapy. Among these 13 patients 5 patients showed complete treatment failure. Higher failure rates may be attributed to the false susceptible results obtained in in-vitro susceptibility testing or to the in-consistent efficacy of drug in in-vitro and in-vivo conditions. In Similar results were reported by Paul et al. [45] where 27\% failure cases in severe MRSA infections treated with vancomycin noted. However, Wunderink et al. [28] reported very high failure rates (64.52\%) in MRSA nosocomial pneumonia patients treated with vancomycin. Besides these, numerous studies have also reported emergence of vancomycin intermediate and vancomycin resistant $S$. aureus isolates. The main reasons attributed or hypothesized for the clinical failures of vancomycin treatment are loss of accessory gene regulator function in MRSA [46] and potential escalation of vancomycin MICs for MRSA [47].

On the other hand FDC therapy (both empirical and shift of therapy) has better efficacy than vancomycin with cure rates of $81.81 \%, 84.84 \%$ and $76.19 \%$ in Group G1B, G2A and G2B patients. Interesting fact to note here is higher efficacy of FDC in G2B patients which were diagnosed with vancomycin resistant MRSA isolate infections. The higher efficacies of FDC may be attributed to various mechanisms through which FDC target various resistance mechanisms in MRSA strains [35]-[39]. However, for the remaining patients which did not respond to the FDC (either empirically/ shift of therapy), FDC + colistin combination therapy was required to achieve the clinical success. The clinical success achieved with an additional cover of colistin indicate the superiority of combination therapy over mono-therapy consisting of combinations of antibacterials having different mode of actions. The use of FDC as an alternative to the vancomycin makes sense not only because of the proved and/ or proposed mechanisms by which it targets the resistant MRSA, but also because of the lack of safe and efficacious alternative to vancomycin. One of the most studied drug, considered as an alternative to vancomycin is linezolid. Despite the apparent advantages of linezolid in the treatment of MRSA infections, concerns about safety often limit its use. Of particular concern is the association of linezolid with serotonin toxicity and thrombocy- 
topenia [48] [49]. The other recommended alternative for vancomycin is clindamycin. However, the use of clindamycin is limited by its bacteriostatic nature and high resistance rates among the MRSA strains. Along with this, the data supporting the use of clindamycin in severe MRSA infections are mostly limited to the paediatric population [50] and older reports describing its efficacy in bone and joint infections [51]. Overall, responses paralleled clinical responses and MRSA eradication at the end of the therapy in empirical FDC treated groups was $30.84 \%$ and $76.19 \%$ greater than empirical vancomycin treated groups in vancomycin, FDC susceptible and vancomycin resistant, FDC susceptible MRSA isolates respectively. Along with better microbiological and clinical responses, there was a significant difference in the complete treatment failure rates in patients treated with these drugs. The complete treatment failure rates in FDC treated patients was significantly low (6.57\%) as compared to vancomycin group (13.51\%). Similar mortality rates (13.60\%) were reported by Stevens et al. [52] in vancomycin treated patients with severe MRSA infections.

\section{Conclusion}

In conclusion, the empirical intravenous FDC therapy seems to be safe and well tolerated, and has higher efficacy than vancomycin in treatment of different pneumonia caused by MRSA. This retrospective study sheds light on an alternative option to use FDC along with colistin to successfully treat the patients which fail to respond to FDC monotherapy.

\section{References}

[1] Rubinstein, E., Kollef, M.H. and Nathwani, D. (2008) Pneumonia Caused by Methicillin-Resistant Staphylococcus aureus. Clinical Infectious Diseases, 46, S378-S385. http://dx.doi.org/10.1086/533594

[2] Hiramatsu, K. and Niederman, M.S. (2005) Health-Care-Associated Pneumonia: A New Therapeutic Paradigm. Chest, 128, 3784-3787. http://dx.doi.org/10.1378/chest.128.6.3784

[3] Kollef, M.H., Shorr, A., Tabak, Y.P., Gupta, V., Liu, L.Z. and Johannes, R.S. (2005) Epidemiology and Outcomes of Health-Care-Associated Pneumonia: Results from a Large US Database of Culture-Positive Pneumonia. Chest, 128, 3854-3862. http://dx.doi.org/10.1378/chest.128.6.3854

[4] American Thoracic Society, Infectious Diseases Society of America (2005) Guidelines for the Management of Adults with Hospital-Acquired, Ventilator-Associated, and Healthcare-Associated Pneumonia. American Journal of Respiratory and Critical Care Medicine, 171, 388-416. http://dx.doi.org/10.1164/rccm.200405-644ST

[5] Micek, S.T., Kollef, K.E., Reichley, R.M., Roubinian, N. and Kollef, M.H. (2007) Health Care-Associated Pneumonia and Community-Acquired Pneumonia: A Single-Center Experience. Antimicrobial Agents and Chemotherapy, 51, 3568-3573. http://dx.doi.org/10.1128/AAC.00851-07

[6] David, M.Z. and Daum, R.S. (2010) Community-Associated Methicillin-Resistant Staphylococcus aureus: Epidemiology and Clinical Consequences of an Emerging Epidemic. Clinical Microbiology Reviews, 23, 616-687. http://dx.doi.org/10.1128/CMR.00081-09

[7] Klein, E. and Smith, D.L. (2007) Laxminarayan R: Hospitalizations and Deaths Caused by Methicillin-Resistant Staphylococcus aureus, United States, 1999-2005. Emerging Infectious Diseases Journal, 13, 1840-1846. http://dx.doi.org/10.3201/eid1312.070629

[8] Jones, R.N. (2010) Microbial Etiologies of Hospital-Acquired Bacterial Pneumonia and Ventilator-Associated Bacterial Pneumonia. Clinical Infectious Diseases, 51, S81-S87. http://dx.doi.org/10.1086/653053

[9] Kawasaki, S., Aoki, N., Kikuchi, H., Nakayama, H., Saito, N., Shimada H., et al. (2011) Clinical and Microbiological Evaluation of Hemodialysis-Associated Pneumonia (HDAP): Should HDAP Be Included in Healthcare-Associated Pneumonia? Journal of Infection and Chemotherapy, 17, 640-645. http://dx.doi.org/10.1007/s10156-011-0228-z

[10] Chastre, J. and Fagon, J.Y. (2002) Ventilator-Associated Pneumonia. American Journal of Respiratory and Critical Care Medicine, 165, 867-903. http://dx.doi.org/10.1164/ajrccm.165.7.2105078

[11] Tablan, O.C., Anderson, L.J., Besser, R., Bridges, C. and Hajjeh, R. (2004) CDC; Healthcare Infection Control Practices Advisory Committee. Guidelines for Preventing Health-Care-Associated Pneumonia, 2003: Recommendations of CDC and the Healthcare Infection Control Practices Advisory Committee. MMWR Recommendations and Reports, 53, $1-36$.

[12] Craven, D.E. and Steger, K.A. (1996) Nosocomial Pneumonia in Mechanically Ventilated Adult Patients: Epidemiology and Prevention in 1996. Seminars in Respiratory Infections, 11, 32-53.

[13] Weinstein, R.A. (1991) Epidemiology and Control of Nosocomial Infections in Adult Intensive Care Units. American Journal of Medicine, 91, 179S-184S. http://dx.doi.org/10.1016/0002-9343(91)90366-6 
[14] Torres, A., Gatell, J.M., Aznar, E., El-Ebiary, M., Puig de la Bellacasa, J., Gonzalez, J., et al. (1995) Re-Intubation Increases the Risk of Nosocomial Pneumonia in Patients Needing Mechanical Ventilation. American Journal of Respiratory and Critical Care Medicine, 152, 137-141. http://dx.doi.org/10.1164/ajrccm.152.1.7599812

[15] Klevens, R.M., Edwards, J.R., Richards Jr., C.L., Horan, T.C., Gaynes, R.P., Pollock, D.A., et al. (2007) Estimating Health Care-Associated Infections and Deaths in U.S. Hospitals, 2002. Public Health Reports, 122, 160-166.

[16] Shorr, A.F., Tabak, Y.P., Gupta, V., Johannes, R.S., Liu, L.Z. and Kollef, M.H. (2006) Morbidity and Cost Burden of Methicillin-Resistant Staphylococcus aureus in Early Onset Ventilator-Associated Pneumonia. Critical Care, 10, R97. http://dx.doi.org/10.1186/cc4934

[17] Shorr, A.F., Haque, N., Taneja, C., Zervos, M., Lamerato, L., Kothari, S., et al. (2010) Clinical and Economic Outcomes for Patients with Health Care Associated Staphylococcus aureus Pneumonia. Journal of Clinical Microbiology, 48, 3258-3262. http://dx.doi.org/10.1128/JCM.02529-09

[18] Abramson, M.A. and Sexton, D.J. (1999) Nosocomial Methicillin-Resistant and Methicillin-Susceptible Staphylococcus aureus Primary Bacteremia: At What Costs? Infection Control \& Hospital Epidemiology, 20, 408-411. http://dx.doi.org/10.1086/501641

[19] Carbon, C. (1999) Costs of Treating Infections Caused by Methicillin-Resistant Staphylococci and Vancomycin-Resistant Enterococci. Journal of Antimicrobial Chemotherapy, 44, 31-36. http://dx.doi.org/10.1093/jac/44.suppl 1.31

[20] Rubin, R.J., Harrington, C.A., Poon, A., Dietrich, K., Greene, J.A. and Moiduddin, A. (1999) The Economic Impact of Staphylococcus aureus Infection in New York City Hospitals. Emerging Infectious Diseases Journal, 5, 9-17. http://dx.doi.org/10.3201/eid0501.990102

[21] Li, J., Willke, R., Pinto, L., Rittenhouse, B.E., Rybak, M.J., Pleil, A.M., et al. (2001) Comparison of Length of Hospital Stay for Patients with Known or Suspected Methicillin-Resistant Staphylococcus Species Infections Treated with Linezolid or Vancomycin: A Randomized, Multicenter Trial. Pharmacotherapy, 21, 263-274. http://dx.doi.org/10.1592/phco.21.3.263.34198

[22] Houck, P.M., Bratzler, D.W., Nsa, W., Ma, A. and Bartlett, J.G. (2004) Timing of Antibiotic Administration and Outcomes for Medicare Patients Hospitalized with Community-Acquired Pneumonia. Archives of Internal Medicine, 164, 637-644. http://dx.doi.org/10.1001/archinte.164.6.637

[23] Rello, J., Molano, D., Villabon, M., Reina, R., Rita-Quispe, R. and Previgliano, I. (2013) Differences in Hospital- and Ventilator-Associated Pneumonia Due to Staphylococcus aureus (Methicillin-Susceptible and Methicillin-Resistant) between Europe and Latin America: A Comparison of the EUVAP and LATINVAP Study Cohorts. Medicina Intensiva, 37, 241-247. http://dx.doi.org/10.1016/j.medin.2012.04.008

[24] Rivera, A.M. and Boucher, H.W. (2011) Current Concepts in Antimicrobial Therapy against Select Gram-Positive Organisms: Methicillin-Resistant Staphylococcus aureus, Penicillin-Resistant Pneumococci, and Vancomycin-Resistant Enterococci. Mayo Clinic Proceedings, 86, 1230-1242. http://dx.doi.org/10.4065/mcp.2011.0514

[25] Gonzalez, C., Rubio, M., Romero-Vivas, J., Gonzalez, M. and Picazo, J.J. (1999) Bacteremic Pneumonia Due to Staphylococcus aureus: A Comparison of Disease Caused by Methicillin-Resistant and Methicillin-Susceptible Organisms. Clinical Infectious Diseases, 29, 1171-1177. http://dx.doi.org/10.1086/313440

[26] Rodvold, K.A. and McConeghy, K.W. (2014) Methicillin-Resistant Staphylococcus aureus Therapy: Past, Present, and Future. Clinical Infectious Diseases, 58, S20-S27. http://dx.doi.org/10.1093/cid/cit614

[27] Bodi, M., Ardanuy, C. and Rello, J. (2001) Impact of Gram-Positive Resistance on Outcome of Nosocomial Pneumonia. Critical Care Medicine, 29, N82-N86. http://dx.doi.org/10.1097/00003246-200104001-00005

[28] Wunderink, R.G., Rello, J., Cammarata, S.K., Croos-Dabrera, R.V. and Kollef, M.H. (2003) Linezolid vs Vancomycin: Analysis of Two Double-Blind Studies of Patients with Methicillin-Resistant Staphylococcus aureus Nosocomial Pneumonia. Chest, 124, 1789-1797.

[29] Hiramatsu, K., Aritaka, N., Hanaki, H., Kawasaki, S., Hosoda, Y., Hori, S., et al. (1997) Dissemination in Japanese Hospitals of Strains of Staphylococcus aureus Heterogeneously Resistant to Vancomycin. The Lancet, 350, 1670-1673. http://dx.doi.org/10.1016/S0140-6736(97)07324-8

[30] Ariza, J., Pujol, M., Cabo, J., Pena, C., Fernandez, N., Linares, J., et al. (1999) Vancomycin in Surgical Infections to Methicillin-Resistant Staphylococcus aureus with Heterogenous Resistance to Vancomycin. The Lancet, 353, 1587 1588. http://dx.doi.org/10.1016/S0140-6736(99)01017-X

[31] Sieradzki, K., Villari, P. and Tomasz, A. (1998) Low-Level Teicoplanin Resistance and Heteroresistance to Vancomycin. Annals of Internal Medicine, 128, 245. http://dx.doi.org/10.7326/0003-4819-128-3-199802010-00021

[32] Sieradzki, K., Roberts, R.B., Haber, S.W. and Tomasz, A. (1999) The Development of Vancomycin Resistance in a Patient with Methicillin-Resistant Staphylococcus aureus Infection. The New England Journal of Medicine, 340, $517-$ 523. http://dx.doi.org/10.1056/NEJM199902183400704

[33] Wong, S.S.Y., Ho, P.L., Woo, P.C.Y. and Yuen, K.Y. (1999) Bacteremia Caused by Staphylococci with Inducible 
Vancomycin Heteroresistance. Clinical Infectious Diseases, 29, 760-767. http://dx.doi.org/10.1086/520429

[34] Centers for Disease Control and Prevention (1997) Update: Staphylococcus aureus with Reduced Susceptibility to Vancomycin-United States. Morbidity and Mortality Weekly Report, 46, 813-815.

[35] Chaudhary, M. and Payasi, A. (2013) Inhibition of in Vitro mecA Gene Transfer among Indian Clinical Isolates of Staphylococus aureus. The Journal of Applied Biochemistry, 106, 133-139.

[36] Chaudhary, M., Patnaik, S.K. and Payasi, A. (2014) Evaluation of Different Drugs in Down-Regulation of Efflux Pump Genes Expression in Methicillin-Resistant Staphylococcus aureus Strains. American Journal of Infectious Diseases, 10, 184-189. http://dx.doi.org/10.3844/ajidsp.2014.184.189

[37] Chaudhary, M., Patnaik, S.K. and Payasi, A. (2014) Inhibition of Panton-Valentine Leukocidin Toxin Induced Neutrophil Cell Lysis by Vancoplus in Methicillin-Resistant Staphylococcus aureus Infections. American Journal of Infectious Diseases, 10, 154-163. http://dx.doi.org/10.3844/ajidsp.2014.154.163

[38] Chaudhary, M. and Payasi, A. (2015) Taming Wall Teichoic Acid Multi Drug Resistance in Gram Positive Pathogens. Journal of Chemical and Pharmaceutical Research, 7, 18-23.

[39] Chaudhary, M. and Payasi, A. (2015) Vancoplus Kinetic Study in Vancomycin Resistant Staphylococcus aureus. International Journal of Pharmaceutical Sciences Review and Research, 31, 135-142.

[40] Dalfino, L., Bruno, F., Colizza, S., Concia, E., Novelii, A., Rebecchi, F., et al. (2014) Cost of Care and Antibiotic Prescribing Attitudes for Community-Acquired Complicated Intra-Abdominal Infections in Italy: A Retrospective Study. World Journal of Emergency Surgery, 9, 39. http://dx.doi.org/10.1186/1749-7922-9-39

[41] Gaynes, R. and Edwards, J.R. (2005) Overview of Nosocomial Infections Caused by Gram-Negative Bacilli. Clinical Infectious Diseases, 41, 848-854. http://dx.doi.org/10.1086/432803

[42] Kallen, A.J., Mu, Y., Bulens, S., Reingold, A., Petit, S., Gershman, K., et al. (2010) Health Care-Associated Invasive MRSA Infections, 2005-2008. Journal of the American Medical Association, 304, 641-648. http://dx.doi.org/10.1001/jama.2010.1115

[43] Johnson, A.P., Davies, J., Guy, R., Abernethy, J., Sheridan, E., Pearson, A., et al. (2012) Mandatory Surveillance of Methicillin-Resistant Staphylococcus aureus (MRSA) Bacteraemia in England: The First 10 Years. Journal of Antimicrobial Chemotherapy, 67, 802-809. http://dx.doi.org/10.1093/jac/dkr561

[44] Liu, C., Bayer, A., Cosgrove, S.E., Daum, R.S., Fridkin, S.K. and Gorwitz, R.J. (2011) Clinical Practice Guidelines by the Infectious Diseases Society of America for the Treatment of Methicillin-Resistant Staphylococcus aureus Infections in Adults and Children. Clinical Infectious Diseases, 52, e18-e55. http://dx.doi.org/10.1093/cid/ciq146

[45] Paul, M., Bishara, J., Yahav, D., Goldberg, E., Neuberger, A., Ghanem-Zoubi, N., et al. (2015) Trimethoprim-Sulfamethoxazole versus Vancomycin for Severe Infections Caused by Meticillin-Resistant Staphylococcus aureus: Randomised Controlled Trial. British Medical Journal, 350, h2219. http://dx.doi.org/10.1136/bmj.h2219

[46] Sakoulas, G., Moellering Jr., R.C. and Eliopoulos, G.M. (2006) Adaptation of Methicillin-Resistant Staphylococcus aureus in the Face of Vancomycin Therapy. Clinical Infectious Diseases, 42, S40-S50. http://dx.doi.org/10.1086/491713

[47] Robert, J., Bismuth, R. and Jarlier, V. (2006) Decreased Susceptibility to Glycopeptides in Methicillin-Resistant Staphylococcus aureus: A 20 Year Study in a Large French Teaching Hospital, 1983-2002. Journal of Antimicrobial Chemotherapy, 57, 506-510. http://dx.doi.org/10.1093/jac/dki486

[48] Lawrence, K.R., Adra, M. and Gillman, P.K. (2006) Serotonin Toxicity Associated with the Use of Linezolid: A Review of Postmarketing Data. Clinical Infectious Diseases, 42, 1578-1583. http://dx.doi.org/10.1086/503839

[49] Perry, C.M. and Jarvis, B. (2001) Linezolid: A Review of Its Use in the Management of Serious Gram-Positive Infections. Drugs, 61, 25-51. http://dx.doi.org/10.2165/00003495-200161040-00008

[50] Martinez-Aguilar, G., Hammerman, W.A., Mason Jr., E.O. and Kaplan, S.L. (2003) Clindamycin Treatment of Invasive Infections Caused by Community-Acquired, Methicillin-Resistant and Methicillin-Susceptible Staphylococcus aureus in Children. Pediatric Infectious Disease Journal, 22, 593-598. http://dx.doi.org/10.1097/01.inf.0000073163.37519.ee

[51] Geddes, A.M., Dwyer, N.S., Ball, A.P. and Amos, R.S. (1977) Clindamycin in Bone and Joint Infections. Journal of Antimicrobial Chemotherapy, 3, 501-507. http://dx.doi.org/10.1093/jac/3.5.501

[52] Stevens, D.L., Herr, D., Lampiris, H., Hunt, J.L., Batts, D.H., Hafkin, B., et al. (2002) Linezolid versus Vancomycin for the Treatment of Methicillin-Resistant Staphylococcus aureus Infections. Clinical Infectious Diseases, 34, 14811490. http://dx.doi.org/10.1086/340353 